\title{
Dredge-Up in Asymptotic Giant Branch Stars
}

\author{
N. Mowlavi \\ Observatoire de Genève, CH-1290 Versoix, Switzerland
}

\begin{abstract}
The third dredge-up phenomenon in asymptotic giant branch stars, responsible for the formation of $\mathrm{C}$ stars, is discussed based on detailed evolutionary model calculations. The structural readjustment of the star as dredge-up proceeds is analyzed, and its consequences on dredge-up predictions discussed. The question of how to obtain dredgeup in asymptotic giant branch models is also addressed. It is stressed, in particular, that the modeling of dredge-up requires some sort of extramixing to be applied below the envelope if the local Schwarzschild criterion is used to delimit convective zones.
\end{abstract}

\section{Introduction}

The third dredge-up (3DUP) phenomenon is the process by which the envelope of asymptotic giant branch (AGB) stars ${ }^{1}$ penetrates into the C-rich layers, bringing to the surface the ashes of helium burning nucleosynthesis (see, e.g., Lattanzio \& Forestini in this volume or Mowlavi 1998b for a general review on the structural and chemical evolution of those stars). It constitutes a key issue in our understanding of the chemical abundances observed at the surface of AGB stars. It is responsible, in particular, for the formation of carbon stars. However, despite the efforts of AGB modelers since more than two decades, reliable predictions of 3DUP are still lacking. Many AGB model calculations even fail to reproduce efficiently, if at all, that phenomenon (for example Vassiliadis \& Wood 1993, Wagenhuber \& Weiss 1994, Blöcker 1995, Forestini \& Charbonnel 1997). The predictions of those which do succeed in obtaining it (for example Straniero et al. 1997, Herwig et al. 1997, Mowlavi 1998a), on the other hand, do not agree with each other. This situation is rather confusing even to experts in AGB modeling, and raises some fundamental questions. Why those contradictory predictions? Can we hope AGB modelers to agree one day on the dredge-up predictions? If not, what are the parameters, whether numerical or physical, responsible for the scatter in those predictions? The lively discussions which animated this 191st IAU Symposium around those questions, among others, revealed that they are not trivial to be answered.

\footnotetext{
${ }^{1}$ The term 'envelope' of AGB stars designates the convective region in their interior which extends from about the photosphere down to layers with temperatures higher than several million degrees. It must not be confused with the 'circumstellar envelope' used in the terminology of observers.
} 
The thermal instabilities (called 'pulses') which periodically develop in the He-burning shell of AGB stars provide an adequate stage for the operation of the 3DUP. After such a thermal pulse, the structural readjustment of the star eventually leads to the deepening of the envelope into the H-depleted regions. While this scenario is well known since twenty years, the physics involved in the dredge-up process has not yet been fully discussed. Yet, a good understanding of that process obviously is a necessary prerequisite to the analysis of model predictions. An effort towards that direction has recently been done by Mowlavi (1998a). It is the purpose of this contribution to highlight some of the basic physical considerations which govern the 3DUP process, and to present their expected consequences on 3DUP predictions.

\section{Modeling third dredge-up}

The question of how to obtain dredge-up in AGB models is related to the delicate question of the determination of convection boundaries. From a numerical point of view, the Schwarzschild criterion is used, to my knowledge, in all AGB models published to date (including my own calculations). This is a local criterion, and consequently raises the question of whether to apply extra-mixing or not beyond the convection boundaries. It is a crucial point in the modeling of third dredge-up. According to my calculations, models strictly using the Schwarzschild criterion (i.e. without any extra-mixing beyond the convection boundaries so defined) do not experience dredge-up. Such models are even unphysical, as they lead to the development of a discontinuity in the abundance profiles at the edge of the convective envelope. In contrast, models with extramixing do lead to efficient dredge-up. Using an extra-mixing procedure of some sort seems thus to be a prerequisite to obtain dredge-up in AGB models.

How much extra-mixing should be used could be an important question too. Fortunately, however, the dredge-up characteristics are found to be rather insensitive to the amount of extra-mixing put in the models. That important conclusion obtained in Mowlavi (1998a) is confirmed by model calculations, and can be understood on grounds of simple physical arguments summarized in Sect. 4. This fact enables us to analyze the dredge-up predictions without worrying much about the details of the extra-mixing. It should be stressed, however, that a diffusion algorithm should be used for the mixing of the chemical elements. Not only does it lead to smooth abundance profiles at the $\mathrm{H}-\mathrm{He}$ transition zone, but it also helps to the numerical calculation of the AGB star structure. Indeed, using instantaneous mixing is known to lead to convergence difficulties (Frost \& Lattanzio 1997).

Extra-mixing has also important consequences on the production of ${ }^{13} \mathrm{C}$ in the intershell regions (Herwig et al. 1997), and on the consequent synthesis of ${ }^{19} \mathrm{~F}$ and s-process elements. The yields of those nuclei, however, are expected to depend on the extra-mixing parameters, as stressed in Mowlavi (1999).

The extra-mixing procedure used in the models presented here considers an exponentially decreasing bubble velocity field below the convective envelope, and uses a diffusion algorithm to perform the mixing of chemical elements. More details on those models can be found in Mowlavi (1998a). 


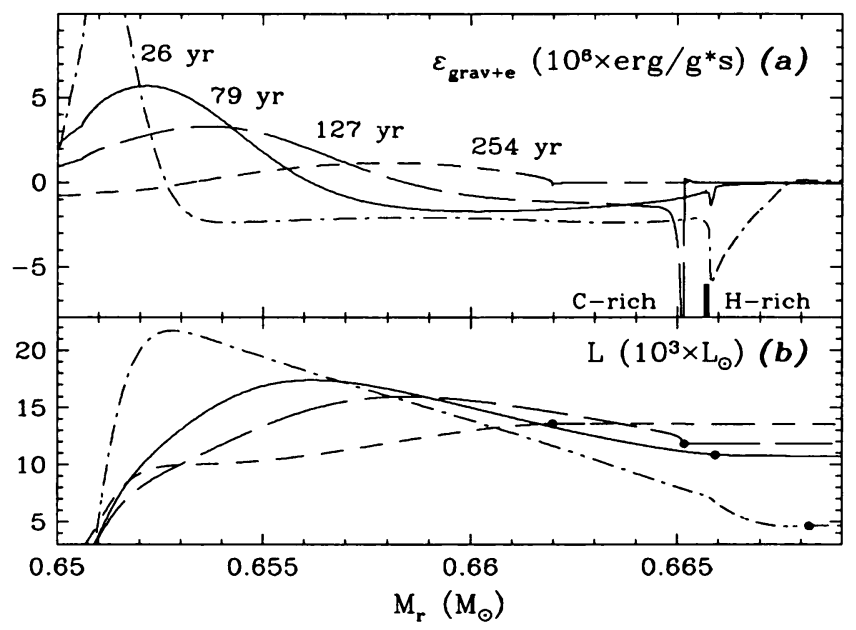

Figure 1. (a) Gravothermal energy profiles at four different times, as labeled on the curves, after the 19 th pulse of a $3 \mathrm{M}_{\odot}$ star computed with extra-mixing below the convective envelope. The time origin is taken at maximum pulse extension. The extents of the $\mathrm{H}$ - and $\mathrm{C}$-rich regions before dredge-up are also indicated, the He-rich region being identified by the thick vertical line separating the $\mathrm{H}$ - and $\mathrm{C}$-rich layers; (b) Same as (a), but for the luminosity profiles. The filled circles on the curves locate the bottom of the convective envelope.

\section{The afterpulse phase}

The afterpulse phase is defined as the phase which follows a thermal pulse and during which the star undergoes various structural and thermal readjustments. Those readjustments result from the outwards evacuation of a positive gravothermal energy which develops at the bottom of the former pulse (Paczyński 1977). In the 19 th afterpulse phase of a $3 \mathrm{M}_{\odot}$ model star, this gravothermal energy wave reaches the $\mathrm{H}$-rich layers in about $250 \mathrm{yr}$ [see Fig. 1(a)]. The luminosity at the bottom of the envelope increases concomitantly with time, reaches a maximum value, and then decreases as the gravothermal energy evacuates outwards [Fig. 1(b)]. The increase in the luminosity around the bottom of the convective envelope renders those layers more unstable against convection, and is responsible for the penetration of the envelope in the deeper layers. Concomitant to the luminosity, the convective envelope reaches a maximum depth, and then retreats back to higher mass locations.

When the envelope reaches the H-depleted layers, a discontinuity in the hydrogen abundance profile develops in models using the Schwarzschild criterion (e.g. Iben 1976). It is this discontinuity that prevents a further deepening of the envelope if no extra-mixing is used (Mowlavi 1998a, 1999). The situation is however unphysical, and a (diffusive) extra-mixing is required as recalled in Sect. 2. 


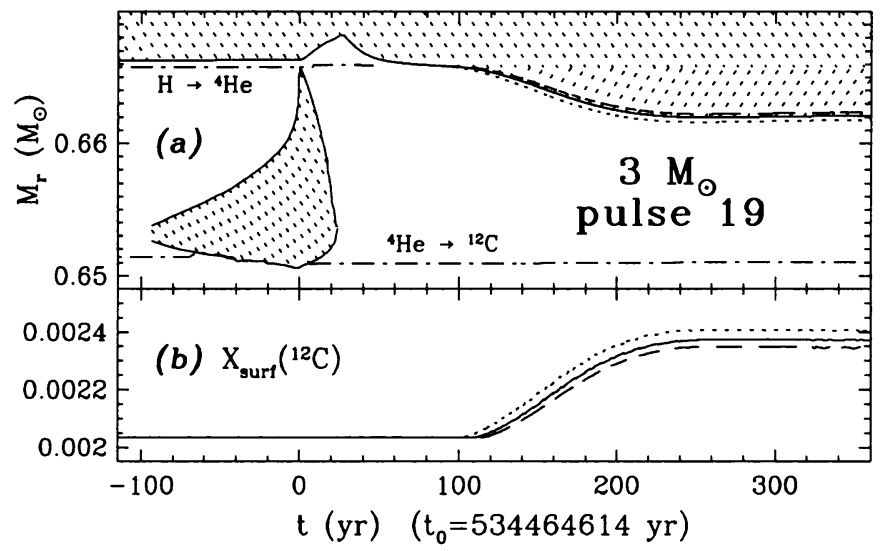

Figure 2. (a) Evolution of the convection zones (shown in hatched) during and after the 19 th pulse in the models of the $3 \mathrm{M}_{\odot}$ star computed with extra-mixing. The solid, dashed and dotted lines differentiate models computed with different extra-mixing parameters (see Mowlavi 1998a). The extent of the overshooting regions is less than $10^{-4} \mathrm{M}_{\odot}$ and cannot be distinguished in the figure; the dashed-dotted lines locate the layer of maximum nuclear energy production in the $\mathrm{H}$ and $\mathrm{He}$ burning shells, as labeled in the graph. The time origin is identical to that in Fig. 1. (b) Same as (a), but for the surface ${ }^{12} \mathrm{C}$ mass fraction.

\section{The third dredge-up process}

Efficient dredge-up is obtained in AGB models using extra-mixing. Model calculations performed with various extra-mixing extents and efficiencies reveal that the dredge-up characteristics are independent of those extra-mixing parameters. Three such sets of calculations are displayed in Fig. 2 for the 19th afterpulse of the $3 \mathrm{M}_{\odot}$ model star. The dredge-up rate is found to be equal to $4.2 \times 10^{-5} \mathrm{M}_{\odot} / \mathrm{yr}$ in all three sets. That the dredge-up rate is insensitive to the extra-mixing parameters can be understood from the following reasoning.

An AGB star can basically be thought, for our purpose, as being composed of a $\mathrm{H}$-depleted core and a $\mathrm{H}$-rich envelope. The core is very dense and compact with a radius of about $0.10-0.15 R_{\odot}$ and a mean density of $10^{5}-10^{7} \mathrm{~g} \mathrm{~cm}^{-3}$. It is for a great part degenerate in the electrons, property which determines the structural evolution of its interior. In contrast, the envelope is rather dilute with a mean density of about $10^{-6} \mathrm{~g} \mathrm{~cm}^{-3}$, and extends up to the photosphere several hundreds of solar radii away from the center of the star (see, e.g., Mowlavi 1995). Convection is its dominant mode of energy transport, and constrains its structure. The envelope structure thus mainly depends on the conditions near the stellar surface rather than on the stellar interior properties.

As dredge-up proceeds, H-depleted matter is added from the core into the envelope. That matter is lifted to higher radius, and its potential energy increases. The characteristic time-scale of that process is determined by the thermal relaxation time-scale of those layers engulfed in the envelope and which must 
expand to fit the envelope structure. This time-scale imposes a maximum rate at which dredge-up can proceed. An estimation of that maximum dredge-up rate, $r_{d u p}$, is given by (see Mowlavi 1998a):

$$
r_{d u p} \simeq 3.2 \times 10^{-8} \frac{R_{c} L_{c}}{M_{c}}
$$

where $M_{c}$ and $R_{c}$ are the mass and radius of the H-depleted core, and $L_{c}$ the luminosity at the core edge. All quantities in Eq. 1 are given in solar units, and $r_{d u p}$ is expressed in $\mathrm{M}_{\odot} / \mathrm{yr}$. For the 19 th pulse of the $3 \mathrm{M}_{\odot}$ star displayed in Fig. $2, M_{c} \simeq 0.66 \mathrm{M}_{\odot}, R_{c} \simeq 0.12 \mathrm{R}_{\odot}$ and $L_{c} \simeq 10^{4} \mathrm{~L}_{\odot}$. Equation 1 then leads to $r_{d u p} \simeq 5.8 \times 10^{-5} \mathrm{M}_{\odot} / \mathrm{yr}$. This is close enough to the value obtained from the evolutionary model calculations.

The mixing time scale of matter across the convection boundary is estimated to be much shorter than the dredge-up rate (Mowlavi 1999). This ensures that the dredge-up rate obtained in AGB models is essentially independent on the extra-mixing parameters. The 3DUP predictions are rather determined by the thermal relaxation time scale of the envelope.

\section{Third dredge-up characteristics}

Equation 1 predicts that the maximum dredge-up rate is proportional to the luminosity at the bottom of the envelope. This is valid to first approximation if we neglect the change in $R_{c} / M_{c}$. If we further assume that the luminosity is constant in the envelope, then we expect a linear relation between the dredge-up rate and the surface luminosity. Dredge-up calculations performed on selected afterpulses of the $3 \mathrm{M}_{\odot}$ star actually confirm that expectation ${ }^{2}$ (Mowlavi 1998a).

The dredge-up efficiency is traditionally expressed by a number $\lambda$, which is the ratio of the amount of matter dredged-up relative to the increase in core mass due to hydrogen burning during two successive pulses. A value of $\lambda=1$, for example, implies a constant core mass from one pulse to the next. Model calculations reveal that $\lambda$, too, increases linearly with the surface luminosity ${ }^{2}$. Moreover, the dredge-up efficiency is predicted to be rather high, reaching unity at $\mathrm{L} \simeq 13000 \mathrm{~L}_{\odot}$ in a $3 \mathrm{M}_{\odot}$ star. High values of $\lambda$ are indeed expected from the analysis of the C-star populations in the Magellanic Clouds (Groenewegen $\&$ de Jong 1993). Our predictions are moreover compatible with those obtained by Herwig et al. (1997) who use an overshooting prescription in their models very similar to ours. The dredge-up efficiencies obtained by those authors are even higher, at equivalent luminosities, than ours. Certainly, the inclusion of extra-mixing in AGB model calculations opens new horizons in the field of AGB modeling.

\footnotetext{
${ }^{2}$ The feedback of the dredge-ups on the evolution of AGB stars, which is not considered in this paper, may alter some 'standard' results of AGB evolution. Such is the case, for example, for the core mass-luminosity relation (Herwig et al. in this volume). The possible consequences of such a feedback on the dredge-up characteristics themselves are discussed in Mowlavi (1998a).
} 


\section{Conclusion}

Obtaining dredge-up, especially in models of low-mass stars, was still a challenge at the beginning of this decade. Sackmann \& Boothroyd (1991) suggested at that time that the solution may reside in the use of new radiative opacities then available, and of higher mixing length parameters for convection. Straniero et al. (1997) even suggest that the difficulty is purely of numerical origin, and that the use of higher spatial and temporal resolution in the models could solve the difficulties of obtaining dredge-up.

The recent studies on the role of extra-mixing in obtaining dredge-up open new perspectives for the study of 3DUP. Herwig et al. (1997) obtain efficient dredge-up in models using extra-mixing. Mowlavi (1998a) further shows that the dredge-up would not occur in AGB models using only the Schwarzschild criterion without any extra-mixing, and that an extra-mixing procedure of some sort is necessary. He shows, moreover, that the dredge-up characteristics are essentially independent of the extra-mixing parameters, provided of course that such an extra-mixing is applied. This allows AGB modelers not to worry too much about the details of the extra-mixing, at least as far as the dredge-up characteristics are concerned. Hopefully, consistent and reproducible predictions of third dredge-up in AGB stars will soon become available.

\section{References}

Blöcker T., 1995, A\&A 297, 727

Forestini M., Charbonnel C., 1997, A\&AS 123, 241

Frost C.A., Lattanzio J.C., 1996, ApJ 344, L25

Groenewegen M.A.T., de Jong T., 1993, A\&A 267, 410

Herwig F., Blöcker T., Schönberner D., El Eid M., 1997, A\&A 324, L81

Iben I., Jr., 1976, ApJ 208, 165

Mowlavi N., 1995, Ph.D. Thesis, Université Libre de Bruxelles ${ }^{3}$

Mowlavi N., 1998a, A\&A, submitted

Mowlavi N., 1998b, eds. M. Arnould et al., in Tours Symposium on Nuclear Physics III, AIP Conf. Proc., New-York, p. $507^{3}$

Mowlavi N., 1999, Nuclei in the Cosmos V, eds. N. Prantzos and S. Harissopulos, Editions Frontières, France, in press ${ }^{3}$

Paczyński B., 1977, ApJ 214, 812

Sackmann I.-J., Boothroyd A.I., 1991, in 'Evolution of Stars: The Photospheric Abundance Connection', IAU Symp. 145, eds. G. Michaud and A. Tutukov, Kluwer Acad. Publ., p. 275

Straniero O., Chieffi A., Limongi M., Busso M., et al., 1997, ApJ 478, 332

Vassiliadis E., Wood P.R., 1993, ApJ 413, 641

Wagenhuber J., Weiss A., 1994, A\&A 286, 121

${ }^{3}$ Postscript file available by anonymous ftp on 'obsftp.unige.ch' in 'pub/mowlavi', or on the WWW at 'http://obswww.unige.ch/ mowlavi/publications'. 